\title{
Referencia de los usos medicinales del chuchuhuasi (Maytenus macrocarpa) por curanderos del distrito de Santa María de Huachipa, Lurigancho, Lima - Perú: un estudio cualitativo
}

Jiménez-Grados Lyane ${ }^{1, a}$; Kolevic-Saraza Niurka ${ }^{1, a}$; Jara-Huancaya Ursula ${ }^{1, a}$; Meléndez-Aquino José ${ }^{1, a}$; Jiménez-Cabezudo Carlos $^{1, a}$; Salazar-Granara Alberto* 1,2,a,b

\section{RESUMEN}

Objetivo: Indagar acerca de las principales indicaciones, propiedades y formas de uso del chuchuhuasi referidas por un grupo de curanderos de Santa María de Huachipa (Lima-Perú). Existen antecedentes de estudios de los usos medicinales del chuchuhuasi (Maytenus macrocarpa) en comunidades nativas de la Amazonía, tanto por el poblador como por indicación del terapeuta tradicional; sin embargo, las referencias del uso y propiedad medicinal del chuchuhuasi en la costa, y en la comunidad urbana son escasas.

Materiales y métodos: Diez curanderos participaron de forma voluntaria. Se aplicó un cuestionario (mediante entrevista) que indagó acerca del uso del chuchuhuasi (propiedades, efectos adversos, contraindicaciones y formas farmacéuticas). La participación se registró con videograbaciones y luego se transcribió; posteriormente, los datos se categorizaron mediante pares y se definieron por consenso.

Resultados: Las principales indicaciones de consumo de chuchuhuasi fueron los problemas respiratorios (80,00 \%) y osteotendinosos. Las propiedades antiinflamatorias y afrodisíacas fueron las más relevantes; y como efecto adverso se reportó el mareo. El producto estaba contraindicado en gestantes, hipertensos y diabéticos. Además, las principales formas farmacéuticas de consumo fueron el macerado, la pomada y la infusión.

Conclusiones: Se encontró la referencia del uso del chuchuhuasi en un grupo de curanderos del distrito de Santa María de Huachipa (Lima-Perú). La indicación más importante de su empleo son los problemas respiratorios y se destaca su propiedad antinflamatoria.

Palabras clave: Maytenus; Medicina tradicional; Antropología cultural; Chamanismo (Fuente: DeCS BIREME)

\section{Information on medicinal uses of chuchuhuasi (Maytenus macrocarpa) from folk healers of the district of Santa María de Huachipa, Lurigancho, Lima - Peru: a qualitative study}

\section{ABSTRACT}

Objective: To inquire the main conditions, properties and ways of consumption of chuchuhuasi from a group of folk healers of the district of Santa María de Huachipa (Lima - Peru). There are studies of the medicinal uses of chuchuhuasi (Maytenus macrocarpa) in native communities of the Amazon known by the inhabitants and indicated by folk healers. However, information on the medicinal uses and properties of chuchuhuasi on the coast and in urban communities is rare.

Materials and methods: Ten folk healers voluntarily participated in the study. An interview questionnaire was administered to inquire the use of chuchuhuasi (properties, adverse effects, contraindications and ways of consumption). The interview was recorded and then transcribed. Afterwards, the data was categorized by peers and defined by consensus.

Results: The main conditions for consuming chuchuhuasi were respiratory tract $(80 \%)$ and osteotendinous junction problems. The most important properties of chuchuhuasi were its anti-inflammatory and aphrodisiac effects. Dizziness was reported as adverse effect. Chuchuhuasi was contraindicated in pregnancy, hypertension and diabetes. In addition, its main ways of consumption were as spirit, ointment and infusion.

Conclusions: Information on the medicinal uses of chuchuhuasi was obtained from a group of folk healers of the district of Santa María de Huachipa (Lima - Peru). The main condition for consuming chuchuhuasi was respiratory tract problems, and its antiinflammatory properties were highlighted.

Keywords: Maytenus; Medicine, traditional; Anthropology, cultural; Shamanism (Source: MeSH NLM).

1 Universidad de San Martín de Porres, Facultad de Medicina Humana, Centro de Investigación de Medicina Tradicional y Farmacología.

La Molina. Lima, Perú.

2 Sociedad Peruana de Farmacología y Terapéutica Experimental (SOPFARTEX), San Borja. Lima, Perú.

a Médico Cirujano.

b Doctor en Medicina.

* Autor corresponsal. 


\section{INTRODUCCIÓN}

Las plantas medicinales han desempeñado y aún desempeñan un papel importante en la cura de enfermedades y dolencias. Muchos de los medicamentos modernos han surgido de la medicina tradicional y enriquecieron los descubrimientos de la química y la bioquímica. Además, en los últimos años, existe una tendencia mundial de retomar el empleo de las plantas medicinales, debido a los numerosos efectos colaterales que ocasionan los medicamentos químicos ${ }^{(1,2)}$.

Por otro lado, el uso tradicional de plantas medicinales sigue siendo una práctica usual en la población; especialmente, en países pluriculturales como el Perú (3,4). En estos casos, disciplinas como la etnomedicina, que estudia el conocimiento tradicional, uso y manejo de las plantas medicinales, ayudan a lograr avances científicos y sociales al integrar prácticas médicas de acuerdo a la cosmovisión de los grupos humanos con el método científico usado por la medicina convencional ${ }^{(5,6)}$. La etnomedicina suele ser un vehículo efectivo de la integración de agentes como los curanderos hacia el sistema de salud; y se ha comprobado que, en su trabajo empírico, se puede encontrar conocimiento valioso, producto de la medicina tradicional, el cual puede ser corroborado y validado por los métodos convencionales, y generar así la articulación entre el conocimiento ancestral y el conocimiento científico ${ }^{(7,8)}$.

En este contexto, el chuchuhuasi, Chuchuwasha o Chuchasha es una especie vegetal de la que se refieren usos medicinales por los pobladores nativos de las comunidades ubicadas en la Amazonía tropical de Perú, Bolivia, Colombia, Ecuador y Brasil (9,10). El nombre vulgar es aplicado para la especie y género Maytenus macrocarpa (Ruiz y Pavon), un árbol grande de la familia de las Celastráceas, que presenta ramas verticiladas, ramitas foliares anguladas, hojas enteras, coriáceas, oblongo-lanceoladas o elípticas, acuminadas, emarginadas y lustrosas en el haz, de 10 a $20 \mathrm{~cm}$ de largo, con inflorescencia axilar, flores diminutas, cáliz colorido, pétalos obovados, blanquecinos, fruto en cápsula ovoide y semillas oblongas con arilo blanco ${ }^{(9,11)}$.

La raíz, corteza y hojas suelen ser utilizadas en maceraciones alcohólicas o acuosas y decocciones, y pueden ser consumidas por vía oral, en copas y vía tópica ${ }^{(8,12)}$. Las enfermedades o eventos para los que se emplea esta especie varían desde los cuadros reumatológicos, lumbalgias, resfriados, bronquitis, enfermedades diarreicas, infecciones parasitarias, hemorroides hasta fisuras en pezones, y también en el tratamiento de la esterilidad femenina y como afrodisíaco ${ }^{(8,12,13)}$.

El distritito de Santa María de Huachipa se creó el 23 de enero de 1992; está considerado como un poblado y pertenece al distrito de Lurigancho, en la provincia de Lima. Alberga migrantes del interior del Perú, y es un distrito mixto con centros agrícolas y zonas urbanas desarrolladas. En este distrito se ubican mercados ambulantes en los que se venden productos agrícolas provenientes de la región central del Perú, se ofrecen servicios de sanación mediante el curanderismo y se expende productos naturales medicinales. El presente estudio reporta los usos medicinales del chuchuhuasi a partir de entrevistas en un grupo de curanderos del distrito de Santa María de Huachipa, Lima-Perú.

\section{MATERIALES Y MÉTODOS}

\section{Diseño y población de estudio}

Este estudio tuvo un diseño etnográfico (cualitativo) y transversal, con un enfoque general de analisis de contenido manifiesto de la comunicación que se obtuvo con curanderos del distrito de Santa María de Huachipa ${ }^{(14)}$. El enfoque específico fue el etnografico, ya que se busco conocer el conocimiento ancestral sobre los usos tradicionales de chuchuhuasi aplicados a la población que acude a consulta con ellos ${ }^{(15)}$. Se capacitó y orientó a los investigadores, que posteriormente recolectaron la información, se alternaron la posición de entrevistador - observador y mantuvieron la reflexividad y actitud neutral ante las posiciones de los entrevistados ${ }^{(16)}$.

El estudio se realizó en el mercado ambulante de Huachipa, ubicado en el cruce de la avenida Las Torres con la avenida Nicolas Ayllón (antes, Carretera Central). Se identifico 15 puestos de oferta de curanderismo y 18 curanderos, a todos se les explicó los objetivos del estudio y se les invitó a participar. De ellos, solo diez aceptaron participar voluntariamente y firmaron el consentimiento informado elaborado para este fin. Cada entrevista duró, aproximadamente, 30 minutos y para la recolección de datos se emplearon registros videográficos y físicos. Las grabaciones fueron transcritas en su totalidad, luego cada investigador estructuró las respuestas de los entrevistados, seguidamente se sometió a debate y finalmente se culminó mediante consenso.

\section{Variables y mediciones}

La recolección de datos se realizó mediante entrevistas orales, mediante un cuestionario validado por expertos, con preguntas abiertas y semiestructuradas, que incluyó las indicaciones de uso, propiedades, efectos adversos, contraindicaciones y formas farmacéuticas del chuchuhuasi.

\section{Consideraciones éticas}

El estudio fue evaluado y aprobado por el Instituto de Investigación de la Facultad de Medicina Humana (FMH) de la Universidad de San Martín de Porres (USMP). Los datos de los participantes solo fueron manejados por el 
Referencia de los usos medicinales del chuchuhuasi (Maytenus macrocarpa) por curanderos del distrito de Santa María de Huachipa, Lurigancho, Lima - Perú: un estudio cualitativo

equipo de investigadores y se preservaron únicamente en las instalaciones del Centro de Investigación de Medicina Tradicional y Farmacología de la FMH-USMP. Así mismo, se aplico el consentimiento informado, se mantuvo la autonomia de los participantes e ingresaron aquellos que entendieron plenamente los objetivos y alcances del estudio. Finalmente, las entrevistas se realizaron en un ambiente seguro y confiable para el participante, y se evitaron los juicios de valor durante este proceso.

\section{RESULTADOS}

Los curanderos entrevistados refirieron alrededor de doce usos del chuchuhuasi, entre los que destacan las afecciones de las vías respiratorias (tos, resfriados y problemas bronquiales), del sistema osteotendinoso (artritis, reumatismo y dolor óseo). Así como también las propiedades antiinflamatorias y afrodisíacas (Tabla 1).

Tabla 1. Usos y propiedades del chuchuhuasi referidas por curanderos del distrito de Santa María de Huachipa (Lima, Perú)

\begin{tabular}{|l|l|}
\hline Uso/propiedad & Encuestados que refieren uso/propiedad \\
\hline Afecciones en las vías respiratorias & 8 \\
\hline Enfermedades osteotendinosas & 5 \\
\hline Alivio del frío & 3 \\
\hline Actividad antiinflamatoria & 3 \\
\hline Actividad como afrodisíaco & 2 \\
\hline Manejo de los cólicos estomacales & 1 \\
\hline Curación de hemorroides & 1 \\
\hline Efecto diurético & 1 \\
\hline Contrarrestar los calambres & 1 \\
\hline Supresión del cólico menstrual & 1 \\
\hline Problemas circulatorios & 1 \\
\hline Contra el dolor muscular & 1 \\
\hline
\end{tabular}

Fuente: Entrevista del estudio

Los entrevistados refirieron conocer tres formas de administración de esta especie vegetal: macerado, pomada e infusión (Tabla 2).

Tabla 2. Formas farmacéuticas de uso del chuchuhuasi referidas por curanderos del distrito de Santa María de Huachipa (Lima, Perú)

\begin{tabular}{|c|c|c|}
\hline Presentación & Preparación & Recomendación de uso \\
\hline Macerado & $\begin{array}{l}\text { Se puede macerar en } \\
\text { agua ardiente de caña, } \\
\text { cañazo y vino }\end{array}$ & $\begin{array}{l}\text { Para potenciar el efecto sobre las vías respiratorias, se añade miel, } \\
\text { jugo de naranja y otras hierbas medicinales. Esto le confiere, } \\
\text { además, un sabor más agradable. }\end{array}$ \\
\hline Pomada & No refieren & $\begin{array}{l}\text { Complementar con el uso de papel periódico caliente y, en casos } \\
\text { de incontinencia urinaria, calentar la zona mediante el uso un } \\
\text { ladrillo caliente. }\end{array}$ \\
\hline Infusión & $\begin{array}{l}\text { Decocción de la corteza } \\
\text { en agua }\end{array}$ & $\begin{array}{l}\text { Como presentación alternativa, se encuentra el chuchuhuasi } \\
\text { pulverizado (polvo) para disolver en agua. }\end{array}$ \\
\hline
\end{tabular}

Fuente: Entrevista del estudio 
Nueve curanderos indicaron no conocer algún efecto adverso producto del consumo de chuchuhuasi. Sin embargo, uno refirió haber conocido de un caso de mareo. En contraste, los entrevistados reportaron conocer algunas contraindicaciones; por ejemplo, cuatro curanderos mencionaron que el chuchuhuasi no debe emplearse en gestantes, al menos en los primeros cinco meses, y que no se puede usar por vía oral en personas que sufren de hipertensión o diabetes mellitus. Adicionalmente, todos los entrevistados refirieron que la dosis para los niños debe ser la mínima, pero no se especifica una cantidad o dosis exacta.

Cuatro curanderos refirieron que los conocimientos acerca del chuchuhuasi los heredaron por la vía familiar, tres mencionaron que lo aprendieron en su comunidad, y tres mencionaron que lo aprendieron en el trabajo. Asimismo, ocho curanderos mencionaron que solo existe un tipo de chuchuhuasi, y un curandero refirió que conocía hasta tres tipos.

\section{DISCUSIÓN}

En la medicina tradicional amazónica, el curandero o chamán, es una persona elegida por sus deidades, comúnmente, surgen de forma patrilineal, y el conocimiento es transmitido de generación en generación ${ }^{(17)}$, en este estudio solo cuatro curanderos de diez, indicaron que recibieron sus conocimientos por vía familiar, los otros seis manifestaron que lo adquirieron por su comunidad y por trabajo. Estudios previos han mostrado que esta conducta se puede deber a que los familiares directos de los curanderos ya no están ligados al uso de medicina tradicional, o no tienen interés en su transmisión o preservación ${ }^{(18,19)}$.

Un estudio en curanderos de la etnia amazónica Bora Bora refiere que existen dos tipos de chuchuhuasi, hembra y macho, y que el macho es el más potente ${ }^{(13)}$. En nuestro trabajo, solo un curandero de Santa María de Huachipa refirió hasta tres tipos de chuchuhuasi, pero no señala ningún detalle con relación a su potencia. En contraste, el mismo estudio ${ }^{(13)}$ refiere que un grupo de curanderos de La Molina (Lima, Perú) mencionó que solo existe un solo tipo de chuchuhuasi, acorde con lo evidenciado por la mayoría de entrevistados en esta investigación.

Los curanderos entrevistados indican la presencia de mareos como un efecto adverso, lo que coincidiría a lo reportado por Matteo y Friso, quienes mencionan el "disconfort físico" como uno de los efectos del consumo tradicional de chuchuhuasi (Maytenus macrocarpa) ${ }^{(20)}$. De la misma forma, este efecto podría tener un sustento biológico en los resultados encontrados en estudios previos en roedores sobre los efectos del chuchuhuasi (Maytenus macrocarpa) en los sistemas nervioso central y cardiovascular en los que causa depresión (21-23).
Los curanderos de Santa María de Huachipa refieren que el chuchuhuasi no debe emplearse en gestantes, por los potenciales efectos teratogénicos, mutagénicos y fetotóxicos de los metabolitos terpenoides reportados en las especies de Matytenus (24-26). Por otra parte, algunos encuestados refirieron no indicar chuchuhuasi en personas con hipertensión o diabetes mellitus. Este hecho no se encuentra acorde con la evidencia científica disponible, ya que estudios previos en modelos animales reportan efectos cardiovasculares depresores $(22,27)$ y metabolitos activos como los flavonoides que presentan comprobados efectos hipoglicemiantes ${ }^{(26,28)}$.

La forma farmacéutica más referida del chuchuhuasi fue el macerado alcohólico, lo que es acorde con lo referido por curanderos de otro distrito en Lima ${ }^{(13)}$. Por otro lado, el estudio de Lock et al. refiere el uso de extractos de la raíz en medicina tradicional ${ }^{(26)}$, y la investigación de Polesna et al. menciona el uso de tinturas y decoctos ${ }^{(29)}$.

Las afecciones de las vías respiratorias fueron el principal uso referido por los curanderos de Santa María de Huachipa. Este hallazgo es coherente con el estudio de Polesna et al. en el que un 69 \% de los curanderos evaluados en cuatro comunidades shipibo-konibo refirieron el uso de chuchuhuasi para resfriados ${ }^{(29)}$. Otros efectos señalados con frecuencia por los curanderos encuestados fueron las propiedades analgésicas y antinflamatorias como ocurre también en el estudio de Huaranca et al. que reportaron, en una comunidad rural de lquitos, usos tradicionales asociados al tratamiento de enfermedades reumatológicas ${ }^{(30)}$. Estas propiedades han sido encontradas en estudios realizados in vivo, en los que mostraron efectos nociceptivos de extractos etanólicos a dosis de $200 \mathrm{mg} / \mathrm{kg}^{(31)}$, y efectos antinflamatorios a $1000 \mathrm{mg} / \mathrm{kg}^{(32)}$.

Una limitación importante de la investigación es la imposibilidad de identificar la familia, el género y la especie de las plantas utilizadas, por lo que el nombre vulgar podría corresponder a otras especies o familias. Por lo tanto, los futuros estudios podrían establecer mecanismos para colectar la especie referida, y complementar el estudio botánico y taxonómico. Asimismo, se considera otra limitación el hecho de que se catalogó a los entrevistados como curanderos y no se ha diferenciado en los diversos agentes tradicionales que se reporta en la medicina tradicional peruana como los herbolarios, los curiosos, los sobadores y los brujos, entre otros. Sería importante realizar estudios de conocimientos terapéuticos en cada uno de ellos y así rescatar los saberes ancestrales.

Se concluye que los curanderos del distrito de Santa María de Huachipa (Lima - Perú) conocen y refieren usos medicinales del chuchuhuasi, principalmente para los problemas respiratorios y las afecciones osteotendinosas, 
Referencia de los usos medicinales del chuchuhuasi (Maytenus macrocarpa) por curanderos del distrito de Santa María de Huachipa, Lurigancho, Lima - Perú: un estudio cualitativo

en los que se ha encontrado un sustento biológico basado en estudios previos.

Agradecimientos: Al Decano de la Facultad de Medicina Humana de la Universidad de San Martín de Porres, Dr. Frank Lizaraso Caparó, por el apoyo y licencias para el desarrollo del presente estudio.

Contribución de autores: Jiménez-Grados Lyane, KolevicSaraza Niurka, Jara-Huancaya Ursula, Meléndez-Aquino José, Jiménez-Cabezudo Carlos, y Salazar-Granara Alberto concibieron la idea y el diseño del estudio; Jiménez-Grados Lyane, Kolevic-Saraza Niurka, Jara-HuancayaUrsula, MeléndezAquino José, Jiménez-Cabezudo Carlos llevaron a cabo la fase de reclutamiento y procesamiento de la información; Jiménez-Grados Lyane, Kolevic-Saraza Niurka, Jara-Huancaya Ursula, Meléndez-Aquino José, Jiménez-Cabezudo Carlos, y Salazar-Granara Alberto realizaron análisis, revisión crítica y redacción de la versión final del artículo.

Fuentes de financiamiento: Este estudio ha sido financiado por los autores y por la Universidad de San Martín de Porres (USMP).

Conflictos de interés: Los autores declaran no tener ningún conflicto de interés.

\section{REFERENCIAS BIBLIOGRÁFICAS}

1. Gurib-Fakim A. Medicinal plants: traditions of yesterday and drugs of tomorrow. Mol Aspects Med. 2006; 27(1): 1-93.

2. Alamgir ANM. Therapeutic use of medicinal plants and their extracts: volume 1: Pharmacognosy. Springer International Publishing; 2017.

3. Organización Mundial de la Salud. Estrategia de la OMS sobre Medicina tradicional 2014-2023 [Internet]. 1a ed. Hong Kong: Organización Mundial de la Salud; 2013. Disponible en: https://apps.who.int/iris/bitstream/ handle/10665/95008/9789243506098_spa.pdf;jsessionid=F38477 07847BFEE402292DABBA0EB7F4? sequence $=1$

4. Mejía Gálvez J, Carrasco ER, Miguel JL, Flores SA. Conocimiento, aceptación y uso de medicina tradicional peruana y de medicina alternativa/complementaria en usuarios de consulta externa en Lima Metropolitana. Rev Peruana Medicina Integrativa. 2017; 2(1): 47.

5. Leonti M, Casu L. Traditional medicines and globalization: current and future perspectives in ethnopharmacology. Front Pharmacol. 2013; 4: 92.

6. Northridge $M E$, Mack R. Integrating ethnomedicine into public health. Am J Public Health. 2002; 92(10): 1561.

7. Maneenoon K, Khuniad C, Teanuan Y, Saedan N, Prom-in S, Rukleng $\mathrm{N}$, et al. Ethnomedicinal plants used by traditional healers in Phatthalung Province, Peninsular Thailand. J Ethnobiol Ethnomed. 2015; 11: 43.

8. Ashu Agbor M, Naidoo S. Ethnomedicinal plants used by traditional healers to treat oral health problems in Cameroon. Evid Based Complement Alternat Med. 2015: 649832.

9. Mejia K, Rengifo E. Plantas medicinales de uso popular en la amazonía peruana. 2a ed. Lima: Agencia Española de Cooperación
Internacional; 2000.

10. Ministerio de Agricultura y Riego del Perú. Diversidad de especies [Internet]. 2015. Disponible en: https://www.minagri.gob.pe/ portal/objetivos/47-sector-agrario/recurso-biodiversidad/345diversidad-de-especies

11. Brack Egg A. Diccionario enciclopédico de plantas útiles del Perú. Programa de las Naciones Unidas para el Desarrollo: Cuzco; 1999.

12. Veloso CC, Soares GL, Pérez AC, Rodrigues VG, Silva FC. Potencial farmacológico de las especies Maytenus y componentes aislados, especialmente tingenona, para el tratamiento de enfermedades inflamatorias dolorosas. Rev Sujetadores Farmacogn. 2017; 27(4): 533-40.

13. Salazar A, Loja B, Rabanal A, Mestanza S, Heringman K, Pinedo D. Comparación de los usos del chuchuhuasi (Maytenus macrocarpa) entre indígenas Bora-Bora de Loreto y chamanes de Lima (Perú). Rev Fitoterapia 2013; 13(1): 61-9.

14. Hsieh H-F, Shannon SE. Three approaches to qualitative content analysis. Qual Health Res. 2005; 15(9): 1277-88.

15. Emerson RM, Fretz RI, Shaw LL. Writing Ethnographic Fieldnotes. 2nd edition. Chicago: University of Chicago Press; 2011.

16. Ng SL, Baker L, Cristancho S, Kennedy TJ, Lingarg L. Qualitative research in medical education. Understanding Medical Education: evidence, theory, and practice. 3rd edition. John Wiley \& Sons, Ltd; 2013.

17. Graña León D. Concepciones sobre la salud en un grupo de curanderos de la selva peruana [Tesis de pregrado]. Lima: Pontificia Universidad Católica del Perú. Facultad de Psicología; 2013.

18. Adekannbi J, Olatokun WM, Ajiferuke I. Preserving traditional medical knowledge through modes of transmission: a postpositivist enquiry. SA J Inform Manag. 2014; 16(1): 9.

19. Maluleka JR, Ngulube P. The preservation of knowledge of traditional healing in the Limpopo province of South Africa. Inform Develop. 2018; 34(5): 515-25.

20. Politi M, Friso F. Amazonian medicinal plants botanical garden of takiwasi center in Peru; a case report of 25 years' hands-on experience. Horticulture Int J. 2018; 2(3): 68-70.

21. Luján-Carpio E, Medina-Salazar H, Mayor-Vega A, MedranoCanchari K, Mazuelos-Rivas M, et al. Anti-Inflammatory and neurobehavioral effects of the leaves from Maytenus macrocarpa (Ruiz and Pavon) briquet in mice. Phcog J. 2019; 11(1): 75-80.

22. Huaccho Rojas JJ, Cavero Aguilar ES, Quezada Rojas MA, Lara Paredes AM, Lluen Escobar SE, Paragulla Bocángel AA, et al. Efectos sobre la temperatura, frecuencia respiratoria, frecuencia cardiaca y electrocardiograma de Maytenus macrocarpa (Ruiz \& Pav.) Briq. (chuchuhuasi). Rev Cubana Plant Med. 2012; 17(3): 233-43.

23. Zambrano C, Zúñiga Espinoza L, Zanabria Puente R, Zegarra Sánchez J, Zaga Quispe N, Pante Medina C, et al. Antipsychotic and behavior effect of the ethanolic extract from the bark of Maytenus macrocarpa (Ruiz \& Pav.) Briq. in mice. Phcog Commn. 2015; 5(4): 244-9.

24. Torpocco V, Chávez H, Estévez-Braun A, Gutiérrez Ravelo A. New dammarane triterpenes from Maytenus macrocarpa. Chem Pharm Bull (Tokyo). 2007; 55(5): 812-4.

25. Chávez H, Rodríguez G, Estévez-Braun A, Ravelo AG, EstévezReyes R, González AG, et al. Macrocarpins A-D, new cytotoxic nor-triterpenes from Maytenus macrocarpa. Bioorg Med Chem Lett. 2000; 10(8): 759-62.

26. Lock O, Perez E, Villar M, Flores D, Rojas R. Bioactive compounds from plants used in peruvian traditional medicine. Nat Prod Commun 2016; 11(3): 315-37.

27. Pamo-Reyna OG. Características de los trabajos publicados sobre 
las propiedades de las plantas en revistas médicas peruanas. Rev Perú Med Exp Salud Pública. 2009; 26(3): 314-23.

28. Abbasi AM, Khan MA, Ahmad M, Zafar M, Jahan S, Sultana S. Ethnopharmacological application of medicinal plants to cure skin diseases and in folk cosmetics among the tribal communities of North-West Frontier Province, Pakistan. J Ethnopharmacol 2010; 128(2): 322-35.

29. Polesna L, Polesny Z, Clavo MZ, Hansson A, Kokoska L. Ethnopharmacological inventory of plants used in Coronel Portillo Province of Ucayali Department, Peru. Pharm Biol. 2011; 49(2): 125-36.

30. Acostupa RJ, Bardales JJ, Teco RM. Uso de las plantas medicinales en la comunidad El Chino, del área de conservación regional comunal Tamshiyacu-Tahuayo, Loreto, Perú. Conocimiento Amazónico. 2016; 4(2): 77-86.

31. Robles-Pizarro V, Tarqui-Cabrera L, Rodríguez-Collazos N, Morales-Chau A, De la Cruz-Manyari J, Ríos-Melgar K, et al. Efecto antinociceptivo del extracto etanólico de las hojas de Maytenus macrocarpa (Ruiz \& Pav.) Briq. "chuchuhuasi" mediante la prueba de contorsiones abdominales en ratones. Horiz Méd. 2014; 14(1): 6-10.

32. León Fernández AM, Tupia Céspedes LL, Turriate Montaldo Y, Maravi Rengifo JE, Barrientos Herrera AJ, Urbano Farje OJ, et al. Evaluación de la actividad analgésica central de las hojas de Maytenus macrocarpa (Ruiz \& Pav.) Briq. (chuchuhuasi). Rev Cubana Plant Med. 2014; 19(4): 349-60.

\section{Correspondencia:}

Alberto Salazar Granara

Dirección: Av. El Corregidor N 1531 , Las Viñas, La Molina. Lima, Perú.

Teléfono: 013652300 extensión 151

Correo electrónico: asalazarg@usmp.pe

\section{Recibido: 01 de junio de 2020}

Evaluado: 14 de agosto de 2020

Aprobado: 25 de agosto de 2020

( $)$ La revista. Publicado por Universidad de San Martín de Porres, Perú.

(c) Br Licencia de Creative Commons Artículo en acceso abierto bajo términos de Licencia Creative Commons Atribución 4.0 Internacional. (http://creativecommons.org/licenses/by/4.0/)

\section{ORCID iDs}

Jiménez-Grados Lyane Kolevic-Saraza Niurka Jara-Huancaya Ursula Meléndez-Aquino José Jiménez-Cabezudo Carlos Salazar-Granara Alberto

\footnotetext{
(1) https: / / orcid.org/0000-0003-1076-4533 (1) https: / / orcid.org/0000-0001-6829-7400 (ㄱ) https: / / orcid.org/0000-0003-0018-3583

(1) https: / / orcid.org/0000-0003-3490-1790

(ㄱ) https://orcid.org/0000-0002-3179-8479

구 https: / / orcid.org/0000-0003-1996-3176
} 